\title{
Varicella-Zoster Virus-associated Fulminant Hepatitis Following Allogeneic Hematopoietic Stem Cell Transplantation for Multiple Myeloma
}

\author{
Hirobumi Saitoh ${ }^{1}$, Naoto Takahashi ${ }^{2}$, Hiroshi Nanjo ${ }^{3}$, Yoshinari Kawabata ${ }^{4}$, \\ Makoto Hirokawa ${ }^{5}$ and Kenichi Sawada ${ }^{2}$
}

\begin{abstract}
Disseminated visceral varicella-zoster virus (VZV) infection rarely occurs in recipients of allogeneic hematopoietic stem cell transplantation (allo-HSCT). To date, only a few cases of isolated VZV-induced fulminant hepatitis following allo-HSCT have been reported. We herein describe the case of a 47-year-old Japanese man with multiple myeloma who developed fulminant hepatitis 17 months after undergoing allo-HSCT. Despite receiving fresh frozen plasma and platelet transfusions, he developed a bleeding tendency (systemic purpura, petechiae and oral bleeding), slipped into a coma and eventually died. He was retrospectively diagnosed with viscerally disseminated VZV infection based on a postmortem examination and multiplex polymerase chain reaction $(\mathrm{PCR})$ amplification.
\end{abstract}

Key words: varicella-zoster virus, fulminant hepatitis, allogeneic hematopoietic stem cell transplantation

(Intern Med 52: 1727-1730, 2013)

(DOI: 10.2169/internalmedicine.52.0118)

\section{Introduction}

Varicella-zoster virus (VZV) reactivation is a frequently observed opportunistic infection that develops after allogeneic hematopoietic stem cell transplantation (allo-HSCT), with a high incidence of $30-50 \%(1,2)$. However, disseminated visceral VZV infection, presenting with ileus, abdominal pain, hepatitis or meningoencephalitis, rarely occurs in recipients of allo-HSCT (3-14). To the best of our knowledge, only a few cases of isolated VZV-induced fulminant hepatitis following allo-HSCT have been reported to date (15-17). We herein report the clinical course of an alloHSCT patient with multiple myeloma who developed fulminant hepatitis 17 months after undergoing allo-HSCT and was diagnosed with viscerally disseminated VZV infection on a postmortem examination.

\section{Case Report}

A 47-year-old Japanese man was diagnosed with Durie/ Salmon stage IIIA IgD- $\lambda$ multiple myeloma. No chromosomal abnormalities were detected on a conventional cytogenetic analysis of the patient's bone marrow. Fluorescent in situ hybridization was not performed. The patient received three courses of vincristine, doxorubicin and dexamethasone therapy, resulting in a partial response. Subsequently, he received high-dose melphalan followed by an infusion of autologous peripheral blood stem cells mobilized with highdose cyclophosphamide and granulocyte colony-stimulating factor. The patient's history of exposure to varicella and zoster was unknown.

Seven months after the autologous transplantation, the patient underwent reduced-intensity stem cell transplantation (RIST) as an institutional trial approved by the Akita University Research Ethics Board. Informed consent was ob-

\footnotetext{
${ }^{1}$ Department of Hematology, Japanese Red Cross Akita Hospital, Japan, ${ }^{2}$ Department of Hematology, Nephrology, and Rheumatology, Akita University Graduate School of Medicine, Japan, ${ }^{3}$ Department of Pathology, Akita University Hospital, Japan, ${ }^{4}$ Department of Hematology, Akita Kumiai General Hospital, Japan and ${ }^{5}$ Cancer Centre, Akita University Hospital, Japan Received for publication January 12, 2013; Accepted for publication March 28, 2013 Correspondence to Dr. Naoto Takahashi, naotot@ doc.med.akita-u.ac.jp
} 
Table. Laboratory Data

\begin{tabular}{|c|c|c|c|c|}
\hline Days after transplantation & Day 521 & Day 523 & Day 524 & Normal Range \\
\hline Platelet count & 27 & 23 & 39 & $117-329 \times 10^{3} / \mu \mathrm{L}$ \\
\hline Aspartate aminotransferase & 59 & 1,872 & 9,600 & $13-33 \mathrm{IU} / \mathrm{L}$ \\
\hline Alanine aminotransferase & 71 & 1,107 & 4,200 & $6-27 \mathrm{IU} / \mathrm{L}$ \\
\hline Lactate dehydrogenase & 461 & 3,222 & 11,460 & $119-229 \mathrm{IU} / \mathrm{L}$ \\
\hline Alkaline phosphatase & 239 & 403 & 661 & $115-359 \mathrm{IU} / \mathrm{L}$ \\
\hline$\gamma$-glutamyl transpeptidase & 119 & 202 & 293 & $11-47 \mathrm{IU} / \mathrm{L}$ \\
\hline Prothrombin time-international normalized ratio & not performed & 4.94 & not performed & $0.9-1.1$ \\
\hline Activated partial thromboplastin time & not performed & 27.9 & 45.0 & $25.0-35.0 \mathrm{~s}$ \\
\hline Fibrinogen & not performed & 122.8 & 41.8 & $160-360 \mathrm{mg} / \mathrm{dL}$ \\
\hline D-dimer & not performed & 113.3 & 139.5 & $<1.0 \mu \mathrm{g} / \mathrm{mL}$ \\
\hline
\end{tabular}

tained from the patient before performing RIST, according to institutional guidelines. A peripheral blood stem cell graft was obtained from an HLA-matched sibling donor. The conditioning regimen included $30 \mathrm{mg} / \mathrm{m}^{2}$ of fludarabine administered for five days and $4 \mathrm{mg} / \mathrm{kg}$ of busulfan administered for two days. The graft-versus-host disease (GVHD) prophylaxis consisted of cyclosporine and a short course of methotrexate. The patient was administered $1,000 \mathrm{mg}$ /day of acyclovir starting on day -12 before RIST to day 54 after RIST for prophylaxis against herpes simplex virus infection. Engraftment was prompt without the development of acute GVHD, and the dose of cyclosporine was slowly tapered.

The patient developed moderate chronic GVHD involving the skin, lacrimal glands and oral mucosa on day 100, at which point, prednisolone therapy $(0.15 \mathrm{mg} / \mathrm{kg}$ per day) was initiated. On day 471 after RIST, salvage chemotherapy, including doxorubicin and dexamethasone, was initiated due to an increased serum IgD level and multiple new bone lesions. After three cycles of chemotherapy, the serum IgD level decreased from 461.0 (before chemotherapy) to 30.6 $\mathrm{mg} / \mathrm{dL}$. The patient was not administered acyclovir for prophylaxis against herpes simplex virus infection from day 55 after RIST, even at the onset of chronic GVHD or the recurrence of multiple myeloma.

On day 521, the patient complained of general fatigue. Laboratory data indicated mild liver dysfunction; the serum aspartate aminotransferase (AST) and alanine aminotransferase (ALT) levels were $59 \mathrm{IU} / \mathrm{L}$ and $71 \mathrm{IU} / \mathrm{L}$, respectively (Table). On day 522, he complained of epigastralgia and severe back pain. On day 523, he complained of severe abdominal pain; however, no skin or mucosal lesions were noted. The serum AST and ALT levels dramatically increased to $1,872 \mathrm{IU} / \mathrm{L}$ and $1,107 \mathrm{IU} / \mathrm{L}$, respectively. The fibrinogen level decreased to $122.8 \mathrm{mg} / \mathrm{dL}$ with an associated increase in the D-dimer level $(113.3 \mu \mathrm{g} / \mathrm{mL})$. The prothrombin time was prolonged to 27.9 seconds (21.4\%, INR 4.94). Abdominal ultrasound and computed tomography (CT) scans of the chest and abdomen showed unremarkable findings. Although tests for hepatitis B surface antigens and hepatitis $\mathrm{C}$ virus antibodies were negative, the patient was diagnosed with fulminant hepatitis. He then received fresh frozen plasma and platelet transfusions. Despite the administration of these treatments, the laboratory data continued to indicate severe liver dysfunction, with AST, ALT and fibrinogen levels of 9,600 IU/L, 4,200 IU/L and $41.8 \mathrm{mg} / \mathrm{dL}$, respectively. The patient developed a bleeding tendency (systemic purpura, petechiae and oral bleeding), slipped into a coma with hyperammonemia $(145 \mathrm{mM})$ on day 524 and died on day 525. Neither brain CT nor lumbar puncture were performed. Multiplex polymerase chain reaction (PCR) amplification for human herpes viruses in a blood sample obtained on day 524 retrospectively revealed the presence of VZV DNA. With the consent of the patient's family, an autopsy of the liver was performed. The liver exhibited extensive foci of necrosis throughout the parenchyma, and an immunohistopathological examination revealed most hepatic cells to be positive for VZV (Figure).

\section{Discussion}

In the present case, VZV infection was retrospectively confirmed on a pathological examination and multiplex PCR analysis. To our knowledge, only a few cases of isolated VZV fulminant hepatitis developing after allo-HSCT have been reported to date (15-17), and disseminated visceral VZV infection presenting with ileus, abdominal pain, hepatitis and/or meningoencephalitis rarely occurs in recipients of allo-HSCT (3-14). The occurrence of GVHD and the duration of the initial antiviral prophylaxis are known to be significant and independent risk factors for the development of VZV after allo-HSCT $(1,18)$, with a pretransplant diagnosis of a lymphoproliferative disorder being another relevant risk factor (2). In the present case, the relapsed myeloma that developed after allo-HSCT led to profound immunodeficiency due to the presence of chronic GVHD and repeated chemotherapy as well as the myeloma itself; thus, the patient was at a high risk for VZV and other opportunistic infections.

Although acyclovir is commonly used during the first year after HSCT for prophylaxis against herpes simplex virus and VZV infection with high efficacy, the appropriate dose and duration of prophylaxis and the point of termination of acyclovir treatment for patients at a high risk for VZV reactivation remain unclear. Prophylaxis has been shown to reduce the incidence of VZV infection only while the administration of such therapy continues, and the incidence of VZV infection at four years post-transplantation is 


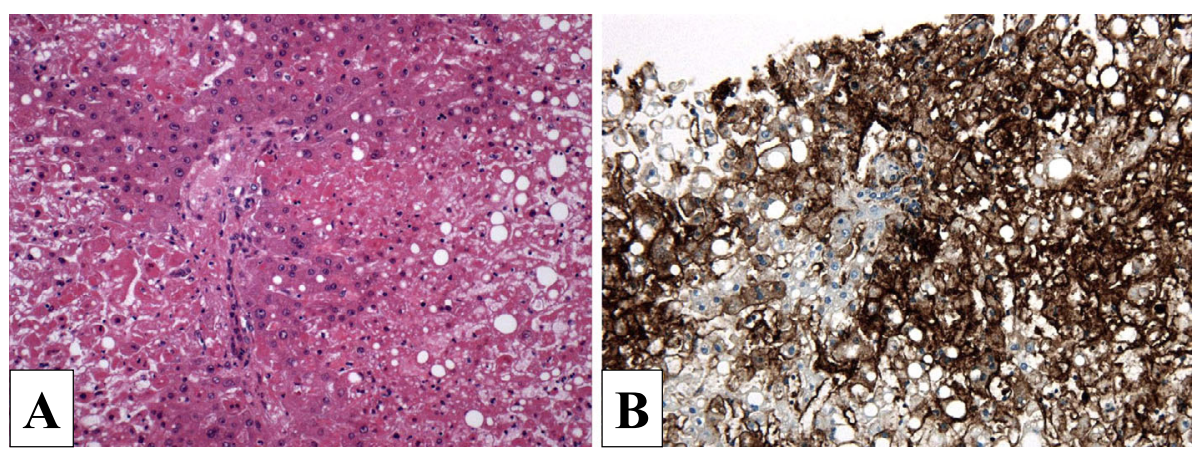

Figure. A. The left panel indicates extensive foci of hepatocyte necrosis throughout the parenchyma. Hematoxylin and Eosin staining; magnification, $\times 100$. B. The right panel indicates the reaction product of staining with anti-varicella-zoster virus (VZV) antibodies (MAB8612, IgG2b, Chemicon, Millipore corporation, Billerica, MA, USA). Immunohistochemical staining; magnification, $\times 100$.

not modified by a 1-year administration of prophylaxis (19). In allo-HSCT recipients with risk factors for VZV infection, long-term prophylactic acyclovir treatment may be required to prevent a fatal VZV infection until the patient's immune system is successfully reconstituted. Thomson et al. attempted to resolve the issue of the appropriate duration of continued VZV prophylaxis after allo-HSCT (20). In their study, HSCT recipients received low-dose acyclovir (400 $\mathrm{mg} /$ day) until all immunosuppressants were discontinued and the CD4 T-cell count exceeded $200 / \mathrm{mm}^{3}$. Although acyclovir prophylaxis prevented early VZV reactivation, the long-term incidence of the disease did not appear to be affected, as infection occurred once prophylaxis was discontinued, even after the CD4 T-cell count exceeded 200/ $\mathrm{mm}^{3}$. In contrast, Asano-Mori et al. reported that long-term prophylaxis consisting of ultra-low-dose acyclovir at $200 \mathrm{mg} /$ day continued until the end of immunosuppressive therapy and for at least one year after transplantation resulted in successful prevention of severe VZV-related symptoms and death, with a significantly decreased overall incidence of VZV reactivation (21). Moreover, with this prophylaxis, visceral dissemination was completely eliminated (21). If our patient had received ultra-low-dose acyclovir prophylaxis until the end of immunosuppressive therapy, it is possible that the fulminant hepatitis could have been prevented.

Recently, the efficacy of 1-year low-dose valacyclovir prophylaxis therapy against VZV infection was prospectively evaluated, wherein visceral involvement and serious complications were completely eliminated for two years after HSCT (22). The inactivated varicella vaccine reduces the risk of VZV reactivation in autologous HSCT patients with lymphoma (23). Although there is controversy surrounding the use of the live attenuated varicella vaccine in allo-HSCT recipients, it is possible that reconstructing the host immunity with the VZV vaccine would reduce the frequency of clinical VZV infection in the period after allo-HSCT. The development of a new prophylactic strategy for treating high-risk patients, including a protocol for VZV vaccination, is needed to prevent fatal VZV infections following alloHSCT.
When VZV disseminates into organs without any cutaneous manifestations, the patient is frequently not treated in a timely manner due to the difficulty in diagnosing the infection. Therefore, disseminated visceral VZV infection, particularly isolated fulminant hepatitis, has an extremely poor prognosis (15-17). Recently, Okamoto et al. reported the successful treatment of severe hepatitis associated with varicella zoster virus infection in a patient with diffuse large B-cell lymphoma treated with rituximab plus combination chemotherapy (24). They initiated treatment with high-dose acyclovir, immunoglobulin and thrombomodulin-alpha and successfully rescued the patient. VZV infection should be considered in patients with unexplained liver dysfunction under severe immunosuppressive conditions, even in the absence of viral exposure and/or skin involvement, and appropriate treatment against VZV infection should be started immediately as empiric therapy due to the high mortality of the disease.

In summary, we herein reported the clinical course of an allo-HSCT patient with multiple myeloma who developed fulminant hepatitis 17 months after undergoing allo-HSCT and who was diagnosed with visceral VZV infection on a postmortem examination. Unfortunately, the patient was not treated in a timely manner due to the difficulty in diagnosing the infection, and he died from VZV infection-induced hepatic failure. In order to successfully treat VZV infections in immunocompromised patients, it is essential to start antiviral therapy with acyclovir empirically when unexplained liver dysfunction occurs after conducting a meticulous physical examination and prompt clinical laboratory tests. The extended use of acyclovir prophylaxis, based on an evaluation of risk factors for VZV, until the end of immunosuppressive therapy should be carefully considered in alloHSCT patients.

The authors state that they have no Conflict of Interest (COI).

\section{References}

1. Koc Y, Miller KB, Schenkein DP, et al. Varicella zoster virus in- 
fections following allogeneic bone marrow transplantation: frequency, risk factors, and clinical outcome: Biology of blood and marrow transplantation. Journal of the American Society for Blood and Marrow Transplantation 6: 44-49, 2000.

2. Kim DH, Messner $\mathrm{H}$, Minden $\mathrm{M}$, et al. Factors influencing varicella zoster virus infection after allogeneic peripheral blood stem cell transplantation: low-dose acyclovir prophylaxis and pretransplant diagnosis of lymphoproliferative disorders. Transpl Infect Dis 10: 90-98, 2008.

3. Teranishi H, Sakiyama M, Nagatoshi $Y$, et al. Acute lymphoblastic leukemia complicated with varicella zoster virus meningoencephalitis and visceral dissemination after related bone marrow transplantation. Jpn J Clin Hematol 52: 287-292, 2011.

4. Peritz DC, Duncan C, Kurek K, Perez-Atayde AR, Lehmann LE. Visceral varicella zoster virus (VZV) after allogeneic hematopoietic stem cell transplant (HSCT) in pediatric patients with chronic graft-versus-host disease (cGVHD). J Pediatr Hematol Oncol 30: 931-934, 2008.

5. Leena M, Ville V, Veli-Jukka A. Visceral varicella zoster virus infection after stem cell transplantation: a possible cause of severe abdominal pain. Scand J Gastroenterol 41: 242-244, 2006.

6. Takatoku M, Muroi K, Kawano-Yamamoto C, Nagai T, Komatsu $\mathrm{N}$, Ozawa K. Involvement of the esophagus and stomach as a first manifestation of varicella zoster virus infection after allogeneic bone marrow transplantation. Intern Med 43: 861-864, 2004.

7. Aoyama Y, Yamamura R, Shima E, et al. Successful treatment with foscarnet for disseminated varicella-zoster infection after reduced intensity stem cell transplantation in a case of relapsed refractory central nervous system lymphoma. Jpn J Clin Hematol 44: 451-455, 2003.

8. Ishizawa J, Fujita H, Iguchi M, Tachibana T, Taguchi J, Ishigatsubo Y. Quantification of circulating varicella-zoster virus DNA for follow-up in a case of visceral varicella-zoster infection ameliorated with intravenous acyclovir. Int J Hematol 85: 242-245, 2007.

9. Kawano N, Gondo H, Kamimura T, et al. Chronic graft-versushost disease following varicella-zoster virus infection in allogeneic stem cell transplant recipients. Int J Hematol 78: 370-373, 2003.

10. Yagi T, Karasuno $T$, Hasegawa $T$, et al. Acute abdomen without cutaneous signs of varicella zoster virus infection as a late complication of allogeneic bone marrow transplantation: importance of empiric therapy with acyclovir. Bone Marrow Transplant 25: 1003-1005, 2000.

11. Akiyama $H$, Inoue $T$, Okoshi $Y$, et al. Varicella-zoster virus infection after hematopoietic stem cell transplantation. Jpn J Clin Hematol 41: 20-24, 2000.

12. Yamazaki E, Kamijoh A, Taguchi J, et al. Fatal acute visceral disseminated varicella-zoster virus infection in a patient with chronic graft-versus-host disease. Jpn J Clin Hematol 45: 1053-1057, 2004.
13. Hamanishi T, Nishikawa $H$, Kobayashi $M$, et al. Acute abdomina pain as a presenting symptom of varicella-zoster virus infection in an allogeneic bone marrow transplant. Jpn J Clin Hematol 39: 5358, 1998.

14. David DS, Tegtmeier BR, O'Donnell MR, Paz IB, McCarty TM. Visceral varicella-zoster after bone marrow transplantation: report of a case series and review of the literature. Am J Gastroenterol 93: 810-813, 1998.

15. Morishita K, Kodo H, Asano S, Fujii H, Miwa S. Fulminant varicella hepatitis following bone marrow transplantation. JAMA 253: 511, 1985.

16. Rogers SY, Irving W, Harris A, Russell NH. Visceral varicella zoster infection after bone marrow transplantation without skin involvement and the use of PCR for diagnosis. Bone Marrow Transplant 15: 805-807, 1995.

17. Perez-Oteyza J, Pascual C, Garcia-Larana J, Odriozola J, Rocamora A, Navarro JL. Abdominal presentation of varicella zoster infection after bone marrow transplantation. Bone Marrow Transplant 9: 217, 1992.

18. Steer CB, Szer J, Sasadeusz J, Matthews JP, Beresford JA, Grigg A. Varicella-zoster infection after allogeneic bone marrow transplantation: incidence, risk factors and prevention with low-dose aciclovir and ganciclovir. Bone Marrow Transplant 25: 657-664, 2000.

19. Boeckh M, Kim HW, Flowers ME, Meyers JD, Bowden RA. Long-term acyclovir for prevention of varicella zoster virus disease after allogeneic hematopoietic cell transplantation--a randomized double-blind placebo-controlled study. Blood 107: 18001805, 2006.

20. Thomson KJ, Hart DP, Banerjee L, Ward KN, Peggs KS, Mackinnon $\mathrm{S}$. The effect of low-dose aciclovir on reactivation of varicella zoster virus after allogeneic haemopoietic stem cell transplantation. Bone Marrow Transplant 35: 1065-1069, 2005.

21. Asano-Mori $Y$, Kanda $Y$, Oshima K, et al. Long-term ultra-lowdose acyclovir against varicella-zoster virus reactivation after allogeneic hematopoietic stem cell transplantation. Am J Hematol 83: 472-476, 2008.

22. Oshima K, Takahashi T, Mori T, et al. One-year low-dose valacyclovir as prophylaxis for varicella zoster virus disease after allogeneic hematopoietic stem cell transplantation. A prospective study of the Japan Hematology and Oncology Clinical Study Group. Transplant infectious disease: an official journal of the Transplantation Society 12: 421-427, 2010.

23. Hata A, Asanuma $\mathrm{H}$, Rinki $\mathrm{M}$, et al. Use of an inactivated varicella vaccine in recipients of hematopoietic-cell transplants. $\mathrm{N}$ Engl J Med 347: 26-34, 2002.

24. Okamoto A, Abe A, Okamoto M, et al. Severe hepatitis associated with varicella zoster virus infection in a patient with diffuse large B cell lymphoma treated with rituximab-CHOP chemotherapy. Int J Hematol 96: 516-520, 2012.

(C) 2013 The Japanese Society of Internal Medicine http://www.naika.or.jp/imonline/index.html 